\title{
PirB inhibits axonal outgrowth via the PI3K/Akt/mTOR signaling pathway
}

\author{
YONG-YAN BI and YONG QUAN \\ Department of Neurosurgery, Minhang Hospital, Fudan University, Shanghai 201199, P.R. China
}

Received April 17, 2016; Accepted September 22, 2017

DOI: $10.3892 / \mathrm{mmr} .2017 .7930$

\begin{abstract}
Accumulating data strongly suggests that leukocyte immunoglobulin like receptor B1 (PirB) inhibits axonal outgrowth. However, the underlying mechanisms remain unclear. In the present study, cortical neurons of newborn mice were cultured with Nogo-66 (Nogo-p4; $4 \mu \mathrm{mol} / \mathrm{l}$; a PirB ligand) together with NEP1-40 (Nogo inhibitory peptide) and/or anti-PirB body $(50 \mathrm{mg} / \mathrm{ml})$. PirB mRNA and protein was higher in cultured neurons induced by Nogo-66 compared with untreated cells. Neurite outgrowth assays demonstrated that the inhibitory effects of Nogo-66 on axonal outgrowth were reversed by anti-PirB body. Reverse transcription-quantitative polymerase chain reaction and western blot assays demonstrated that anti-PirB treatment led to reduced mRNA and protein expression of phosphoinositide 3-kinase (PI3K), Akt serine/threonine kinase (Akt), mechanistic target of rapamycin kinase (mTOR), myosin IIA and cofilin, which are involved in axonal outgrowth. Furthermore, blockade of the PI3K/Akt/mTOR pathway using a PI3K inhibitor or an mTOR inhibitor diminished the stimulatory effect of anti-PirB on axonal outgrowth, and the reduced effect of anti-PirB on factors that were activation by anti-PirB. In addition, blockade of PI3K/Akt/mTOR enhanced anti-PirB-induced gene and protein expression. These results revealed that PirB functions as a potential suppressor in axonal outgrowth via repressing PI3K/Akt/mTOR signaling pathway, and PirB/PI3K/Akt/mTOR may be a novel target for enhancing axonal outgrowth for developing rational therapeutic strategies.
\end{abstract}

\section{Introduction}

Axonal regeneration in the central nervous system (CNS) of adult mammals is very limited, leading to poor functional

Correspondence to: Professor Yong Quan, Department of Neurosurgery, Minhang Hospital, Fudan University, 170 Xin-Song Road, Shanghai 201199, P.R. China

E-mail: quan201616@126.com

Key words: leukocyte immunoglobulin like receptor B1, phosphoinositide 3-kinase/Akt serine/threonine kinase/mechanistic target of rapamycin kinase, axonal outgrowth, neuron recovery after CNS injury (1). In addition, effective ways for promoting the regeneration of injured neurons have not been developed yet (2). Therefore, novel strategies for enhancing axonal outgrowth after injury are urgently required. CNS axons have regeneration capacity, but fail to regenerate predominantly due to environment obstacles that inhibit axonal regeneration after injury (3). It is reported that the inhibitory activity is closely associated with CNS myelin components and other molecules at the injury site (4). The three myelin-derived inhibitors, reticulon 4 (Nogo), myelinassociated glycoprotein (MAG) and oligodendrocyte myelin glycoprotein (OMgp), are potential inhibitors of axonal outgrowth in vitro; they interact with the lycosylphosphatidylinositol-anchored Nogo receptor $(\mathrm{NgR})$ via their C-terminal 66-residue loops (Nogo-66) (5). The inhibitory activities of MAG, OMgp and the extracellular domain of Nogo-A (Nogo-66) on regeneration may be regulated by common receptor complexes, which consist of $\mathrm{NgR}$ and its signaling coreceptors (6). Genetic deletion of $\mathrm{NgR}$ only exerted a modest disinhibitory effect, and the axonal regeneration was partially blocked in neurons of NgR-null mice (7). Similar to $\mathrm{NgR}$, leukocyte immunoglobulin like receptor B1 (PirB) functions as a receptor of Nogo-66, MAG and OMgp (8). The combined inhibition of $\mathrm{NgR}$ and PirB contributes to an almost complete regeneration of damaged nerves (9). PirB, as a functional receptor, is also capable of inhibiting neurite outgrowth $(8,10)$. However, the molecular mechnisms underlying the signaling that inhibits axonal outgrowth through PirB needs to be identified.

Phosphoinositide 3-kinase (PI3K)/Akt serine/threonine kinase (Akt)/mechanistic target of rapamycin kinase (mTOR) signaling pathway has a critical role in regulating axonal outgrowth of mammals (11). Upregulation of mTOR signaling promotes axon regeneration (12). Considering that the importance of $\mathrm{PI} 3 \mathrm{~K} / \mathrm{Akt} / \mathrm{mTOR}$ in regulation of axonal outgrowth, we hypothesized that inhibition of PirB stimulates axonal outgrowth via PI3K/Akt/mTOR signaling pathway.

To the best of our knowledge, the present study is the first to investigated the effects of PirB on axonal outgrowth via $\mathrm{PI} 3 \mathrm{~K} / \mathrm{Akt} / \mathrm{mTOR}$ signaling. The findings may provide a strategy to develop novel therapeutics that enhance regeneration.

\section{Materials and methods}

Animals. BALB/c mice (1-day-old) obtained from the Shanghai Experimental Animal Center (Shanghai, China) were used in 
the experiments. Protocols were followed by the Animal Care and the Guidelines of Animal Use and Protection of Fudan University. The study was approved by the ethics committee of Minhang Hospital, Fudan University (Shanghai, China). Every effort was made to minimize the suffering of animals.

Isolation, culture and identification of cortical neurons. Mice (1-day-old) were sacrificed by cervical dislocation. The cortices were isolated and transferred in an ice-cold calciumand magnesium-free Hank's balanced salt solution. The tissues were digested with $0.125 \%$ trypsin for $20 \mathrm{~min}$, and were washed three times with Dulbecco's minimal essential media (DMEM; $10 \mathrm{ml}$ ) with $20 \%$ fetal bovine serum (Gibco; Thermo Fisher Scientific, Inc, Waltham, MA, USA). The cells were suspended at $1.5 \times 10^{5}$ cells $/ \mathrm{cm}^{2}$ in DMEM supplemented with $10 \%$ horse serum, $10 \%$ fetal bovine serum, $2 \mathrm{mM}$ glutamine and $25 \mathrm{mM}$ glucose, and cultured in a humidified $5 \% \mathrm{CO}_{2}$ incubator at $37^{\circ} \mathrm{C}$. On the second day, $10 \mu \mathrm{M}$ cytarabine (Ara-C) was added to the cultures to inhibit non-neuronal cell proliferation. Twice a week, half of the medium was replaced with fresh fetal bovine serum-free medium. The experiments on the cultured neurons were performed after 10 days. Nogo inhibitory peptide (NEP1-40; Merck KGaA, Darmstadt, Germany), a Nogo-66 receptor antagonist peptide with sequence 1-40 amino acids, acts as the competitive antagonist of $\mathrm{NgR}$ and blocks Nogo-66 inhibition of axonal outgrowth in vitro (9). A total of $4 \mu \mathrm{mol} / \mathrm{l}$ NEP1-40 was added at the time of cell plating, and cultures were incubated for $2 \mathrm{~h}$ before anti-PirB antibody treatment. The neurons were randomly divided into two groups: Negative control and anti-PirB antibody treatment. For the anti-PirB antibody treatment group, the cells $\left(1.5 \times 10^{5}\right.$ cells $\left./ \mathrm{cm}^{2}\right)$ were incubated in Earle's culture medium (Gibco; Thermo Fisher Scientific, Inc.) supplemented with $50 \mu \mathrm{g} / \mathrm{ml} 6 \mathrm{C} 1$, a specific anti-PirB antibody (Santa Cruz Biotechnology, Inc., Dallas, TX, USA) binding the PirB extracellular region, and incubated with a PI3K inhibitor (LY294002, $50 \mu \mathrm{mol} / \mathrm{l})$ and an mTOR inhibitor (rapamycin, $100 \mathrm{nmol} / \mathrm{l}$ ) after $4 \mathrm{~h}$.

Neurite outgrowth assay. The cultured cortical neurons $\left(2 \times 10^{4}\right)$ were cultured on poly-L-lysine/laminin (PLL; a substrate for neuronal outgrowth)-coated 6-well plates with or without purified myelin, NEP1-40 and anti-PirB $(8,13)$. At 72-h post-plating, explants were incubated with Nogo-66 (4 $\mu \mathrm{mol} / 1$; cat no. sc-25659; Santa Cruz Biotechnology, Inc.). The length of the longest neurite for each neuron was determined by systematic scanning of the slide was analyzed by an inverted fluorescence microscope (Olympus Corporation, Tokyo, Japan). Neurite length quantitation was statistically analyzed as described previously (8).

Reverse transcription-quantitative polymerase chain reaction (RT-qPCR). Total RNA was extracted using TRIzol according to the manufacturer's protocol (Invitrogen; Thermo Fisher Scientific, Inc.), and the quality was assessed by spectrophotometric absorbance at 260/280 nm. The extracted RNA was reverse transcribed into cDNA using a RevertAid First Strand cDNA Synthesis kit at $37^{\circ} \mathrm{C}$ for $15 \mathrm{~min}$, followed by $85^{\circ} \mathrm{C}$ for $5 \mathrm{sec}$ (Fermentas; Thermo Fisher Scientific, Inc.). For each sample, $2 \mu \mathrm{g}$ RNA was used to synthesize the cDNA. RT-qPCR was performed with an ABI Prism 7700 sequence detection system (Applied Biosystems; Thermo Fisher Scientific. Inc.). Reactions were performed in duplicate with SYBR Green PCR Master mix (Applied Biosystems; Thermo Fisher Scientific. Inc.). PCR amplification were carried out accorrding to the following program (40 cycles): Primary extension $94^{\circ} \mathrm{C}$ for $5 \mathrm{~min}$; cycling at $94^{\circ} \mathrm{C}$ for $0.5 \mathrm{~min}, 55^{\circ} \mathrm{C}$ for $0.5 \mathrm{~min}$ and $72^{\circ} \mathrm{C}$ for $0.5 \mathrm{~min}$; and final extension at $72^{\circ} \mathrm{C}$ for $10 \mathrm{~min}$. Amplification plots and cycle threshold values from the exponential phase of the PCR were assessed in ABI Prism SDS1.7 software using Genescan version 3.7 software (Applied Biosystems; Thermo Fisher Scientific. Inc.). Only one product of the desired size was identified and one single peak was observed in a melting curve for each primer. Each sample was conducted three times. The relative mRNA expression of each gene was calculated using the $2^{-\Delta \Delta C q}$ method and normalized to $\beta$-actin (14). The primers designed for RT-qPCR are presented in Table I.

Western blot analysis. The protein levels of PirB, PI3k, Akt, cofilin, myosin IIA, mTOR and phosphorylated mTOR were assessed by western blot. Briefly, cultured neurons were harvested in radioimmunoprecipitation assay lysis buffer (Santa Cruz Biotechnology, Inc.). The concentration of protein was determined using bicinchoninic acid protein assay kit (cat no. 23225; Thermo Fisher Scientific. Inc.). Equal amounts of protein $(20 \mu \mathrm{g})$ were denatured for $5 \mathrm{~min}$ at $95^{\circ} \mathrm{C}$ in sample buffer and separated by $10 \%$ sodium dodecyl sulfate-polyacrylamide gel electrophoresis. Following electrophoresis, the proteins were transferred to a polyvinylidene fluoride membrane, which was incubated in blocking solution containing $5 \%$ non-fat milk in a Tris-buffered saline $/ 0.05 \%$ Tween 20 solution for $60 \mathrm{~min}$ at room temperature. Western blot analysis was performed by overnight incubation at $4^{\circ} \mathrm{C}$ using antibodies against PirB (cat no. 03-1647, 1:1,000; EMD Millipore, Billerica, MA, USA), PI3K (cat no. 05-1563, 1:1,000; EMD Millipore), Akt, (cat no. 05-591MG, 1:1,000; EMD Millipore), cofilin (cat no. 07-326, 1:1,000; EMD Millipore), myosin IIA (cat no. MABS755, 1:1,000; EMD Millipore), mTOR (cat no. 05-1592, 1:1,000; EMD Millipore), and phosphorylated mTOR (cat no. 09-345, 1:1,000; EMD Millipore) diluted in $10 \%$ horse serum in TBS-Tween buffer $(0.2 \mathrm{M} \mathrm{NaCl}, 25 \mathrm{mM}$ Tris, $\mathrm{pH} 7.5,0.5 \mathrm{ml} / \mathrm{l}$ Tween-20), followed by incubation with a horseradish peroxidase-coupled mouse secondary antibody (1:10,000, cat no. sc-2004; Santa Cruz Biotechnology, Inc.). Blots were reprobed with $\beta$-actin antibody (cat no. 612656; 1:4,000; BD Bioscience, San Jose, CA, USA), $\alpha$-tubulin III (ABT170; 1:1,000; Merck KGaA) GAPDH (cat no. GB11002-50; 1:1,000; Merck KGaA) at room temperature for $2 \mathrm{~h}$. Signals were analyzed with the Beyotime ECL Plus detection kit (Beyotime Institute of Biotechnology, Haimen, China). Protein were normalized by comparison with the corresponding $\beta$-actin signal of the samples; the data are presented as percentages of the normalized control signal. Densitometric analysis was performed using Image $\mathbf{J}$ software (version 2.1.4.7; National Institutes of Health, Bethesda, MD, USA).

Statistical analysis. All data are presented as the mean \pm standard error. An unpaired t-test and one way-analysis of 
Table I. Primers for qRT-PCR.

\begin{tabular}{|c|c|c|c|c|}
\hline Gene & Sequence $\left(5^{\prime}-3^{\prime}\right)$ & $\operatorname{Tm}\left({ }^{\circ} \mathrm{C}\right)$ & Size (bp) & Locus \\
\hline PI3K & & 60 & 241 & KF011500 \\
\hline $\mathrm{F}$ & ACCCAAGCGAGGATGAGG & & & \\
\hline $\mathrm{R}$ & TGTTGCCCGTGTTGAATG & & & \\
\hline Akt1 & & 60 & 215 & KF011501 \\
\hline $\mathrm{F}$ & TGCTGGATAAAGATGGAC & & & \\
\hline $\mathrm{R}$ & CTGGTTGTAGAAAGGGAG & & & \\
\hline mTOR & & 60 & 93 & KC424580 \\
\hline $\mathrm{F}$ & TCATTTGTTACTACCTCCCA & & & \\
\hline $\mathrm{R}$ & TCTAGAGCAGCTTTGCGAGCCAC & & & \\
\hline Cofilin & & 58.2 & 111 & KC424581 \\
\hline $\mathrm{F}$ & GTTCAGGCTCACCCCGTTCTT & & & \\
\hline $\mathrm{R}$ & AGTAGAGTCATCTGGGCTATCAA & & & \\
\hline Myosin IIA & & 53.9 & 129 & L21170 \\
\hline $\mathrm{F}$ & TTGGTGGAGCGATTTGTC & & & \\
\hline $\mathrm{R}$ & ATCTCGGGTGGCTGAACG & & & \\
\hline$\beta$-actin & & 59.6 & 92 & M26111 \\
\hline $\mathrm{F}$ & CAACGAGCGGTTCAGGTGT & & & \\
\hline $\mathrm{R}$ & TGGAGTTGAAGGTGGTCTCGT & & & \\
\hline
\end{tabular}

F, sense; R, antisense; PI3K, phosphoinositide 3-kinase; Akt1, Akt serine/threonine kinase 1; mTOR, mechanistic target of rapamycin kinase; Tm, melting temperature.

A

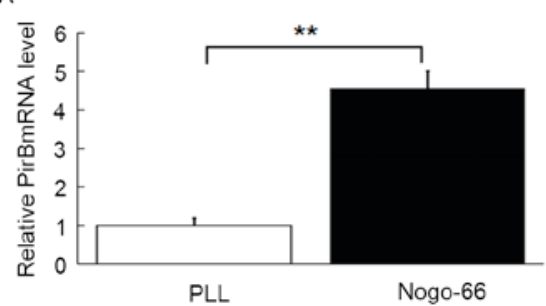

B

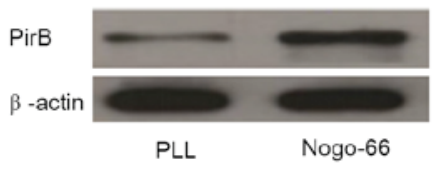

c

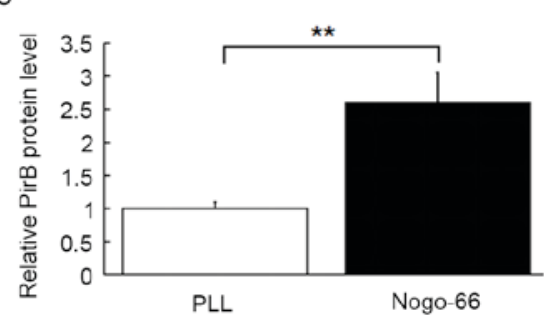

Figure 1. Nogo-66 induces expression of PirB in cortical neurons of newborn mice. (A) PirB mRNA levels were measured by reverse transcription-quantitative polymerase chain reaction. (B) PirB protein levels were assessed by western blot and (C) densitometry analysis was performed. ${ }^{* *} \mathrm{P}<0.01$. PLL, poly-L-lysine/laminin; PirB, leukocyte immunoglobulin like receptor B1.

variance (Bonferroni post hoc test for equal variances assumed; Tamhane's T2 post hoc test for equal variances not assumed) were used to compare the groups using GraphPad Prism version 5.0 software (GraphPad Software, Inc., La Jolla, CA, USA) and SPSS software v.22.0 (IBM Corp., Armonk, NY, USA). $\mathrm{P}<0.05$ was considered to indicate a statistically significant different.

\section{Results}

Nogo-66 induces endogenous PirB expression in cultured neurons. Nogo-66 inhibits axonal outgrowth of cultured neurons through NgR and PirB. NEP1-40 is a Nogo-66 receptor antagonist peptide with the 1-40 amino acid sequence that acts as a competitive antagonist of $\mathrm{NgR}$ and blocks Nogo-66 inhibition of axonal outgrowth in vitro. In order to determine the role of PirB, the cultured neurons were treated with NEP1-40 to block the effect of Nogo-66 on axonal outgrowth. Regulation of PirB mRNA and protein expression by exogenous Nogo-66 was investigated in cultured neurons, treated with NEP1-40. The mRNA levels of PirB were upregulated by treatment with exogenous Nogo-66 compared with cells cultured on PLL (Fig. 1A; $\mathrm{P}<0.01)$. PirB protein levels were also increased by Nogo-66 ( $\mathrm{P}<0.01$; Fig. 1B and $\mathrm{C}$ ). Therefore, Nogo-66 may enhance PirB expression in cultured neurons.

Inactivation of PirB induced axonal outgrowth. To elucidate the role of PirB in axon growth, its influence on axon outgrowth of cultured neurons was investigated. No marked difference in the axonal length of neurons cultured on PLL was observed when neurons were cultured in the presence of NEP1-40, anti-PirB (50 mg/ml) or NEP1-40 + anti-PirB (Fig. 2). The axonal length of neurons was decreased when they were placed on growth-non permissive (Nogo-66) substrate. $(70 \pm 4 \mu \mathrm{m}$ on Nogo-66 compare with $121 \pm 10$ on PLL; $\mathrm{P}<0.01)$. NEP1-40 or anti-PirB treatment partially 

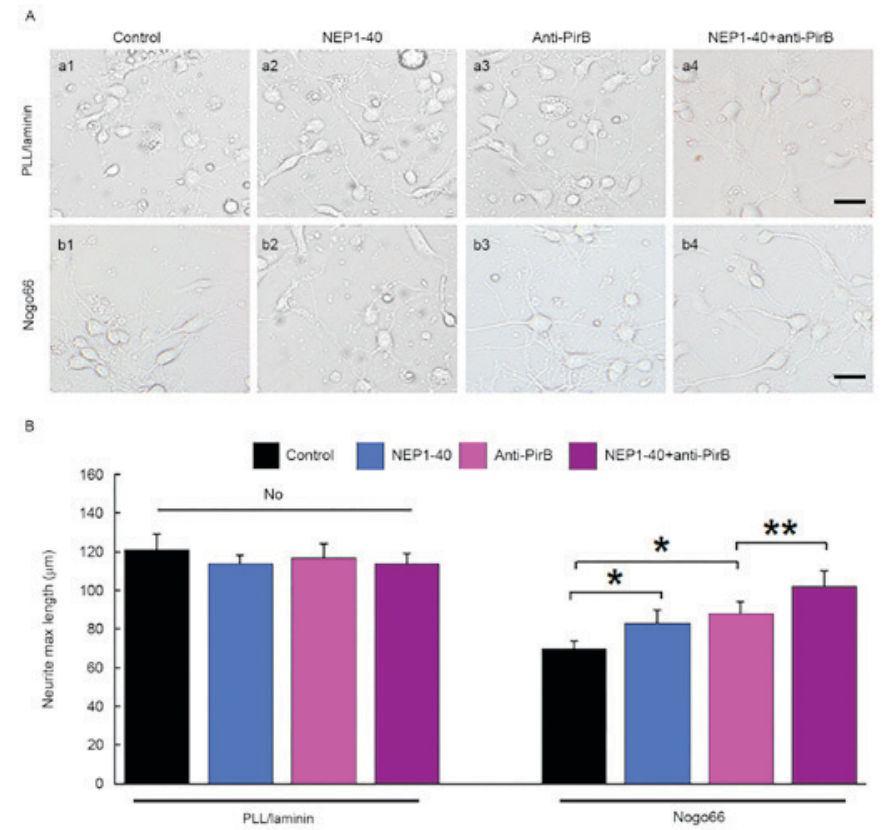

Figure 2. Anti-PirB mediated-axonal outgrowth on non-permissive substrate. Cultured neurons were placed on PLL (control) or Nogo-66 to test inhibition by these substrates after various manipulations. (A) Cultured neurons were incubated on PLL or Nogo-66, either alone or treated with NEP1-40 and/or anti-PirB $(50 \mathrm{mg} / \mathrm{ml})$. (B) Average neurite length was analyzed. ${ }^{*} \mathrm{P}<0.05$; ${ }^{* *} \mathrm{P}<0.01$. Scale bar, $20 \mathrm{~mm}$. PLL, poly-L-lysine/laminin; NEP1-40, Nogo inhibitory peptide; PirB, leukocyte immunoglobulin like receptor B1.

attenuated the inhibitory effect of Nogo-66 and increased the axon length on non-permissive substrate $83 \pm 7$ and $88 \pm 6 \mu \mathrm{m}$, respectively, compared with $70 \pm 4 \mu \mathrm{m}$ with Nogo66 only; $\mathrm{P}<0.05$; Fig. 2). NEP1- $40+$ anti-PirB treatment almost fully attenuated the inhibitory effect of Nogo66 on axon length, and increased the length compared with NEP1-40 or anti-PirB treatment alone $(115 \pm 11 \mu \mathrm{m} ; \mathrm{P}<0.01)$ on non-pemissive substrate (Fig. 2).

Inactivation of PirB activated PI3K/Akt/mTOR signaling pathway. To study whether the PI3K/Akt/mTOR pathway is involved in axonal outgrowth in the presence of anti-PirB, the mRNA levels of the associated genes, including PI3K, Akt1, mTOR, cofilin and myosin IIA, and phosphorylation of mTOR were detected by RT-qPCR and western blot. As shown in Fig. 3A, anti-PirB treatment $(50 \mu \mathrm{g} / \mathrm{ml})$ significantly increased the expression of PI3K, Akt1, Akt2, mTOR, cofilin and myosin IIA mRNA in the NEP1-40 + anti-PirB group compared with the NEP1-40 group $(\mathrm{P}<0.05)$. Anti-PirB treatment promoted the phosphorylation of PI3K, Akt and mTOR, and enhanced cofilin and myosin IIA protein expression $(\mathrm{P}<0.05$; Fig. 3B-D). These results indicated that the $\mathrm{PI} 3 \mathrm{~K} / \mathrm{Akt} / \mathrm{mTOR}$ pathway may mediate PirB-induced inhibition of neurite outgrowth. No significant difference was found between the AP-Nogo66 and AP-Nogo-66 + NEP1-40 groups (data not shown).

Blockade of PI3K/Akt/mTOR pathway inhibits Anti-PirBinduced axonal outgrowth. To further explore the role of the $\mathrm{PI} 3 \mathrm{~K} / \mathrm{Akt} / \mathrm{mTOR}$ pathway in anti-PirB-mediated promotion of neurite outgrowth, two pharmacological inhibitors, a PI3K inhibitor (LY294002) and an mTOR inhibitor (rapamycin),

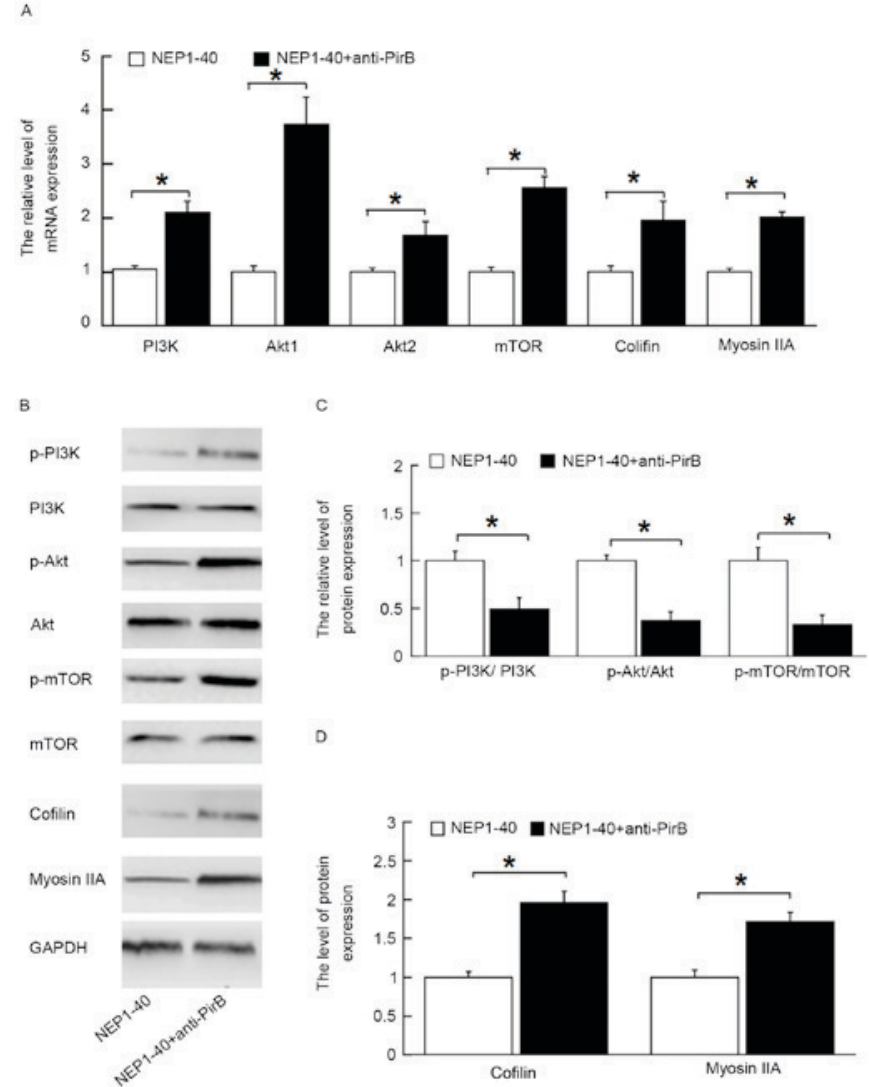

Figure 3. Effect of anti-PirB on axonal outgrowth induced by Nogo66 is associated with to the regulation of PI3K/Akt/mTOR pathways. (A) Reverse transcription-quantitative polymerase chain reaction analysis of selected genes from the PI3K/Akt/mTOR pathway. (B) Western blot analysis of $\mathrm{PI} 3 \mathrm{~K} / \mathrm{Akt} / \mathrm{mTOR}$ signals. Densitomentric analysis of western blots was performed, with quantification relative to GAPDH. (C) Protein phosphorylation levels of PI3K, Akt and mTOR. (D) Protein expression levels of cofilin and myosin IIA. "P<0.05. NEP1-40, Nogo inhibitory peptide; PirB, leukocyte immunoglobulin like receptor B1; PI3K, phosphoinositide 3-kinase; Akt, Akt serine/threonine kinase; mTOR, mechanistic target of rapamycin kinase.

were used in the neurite outgrowth assay. Both LY294002, and rapamycin significantly attenuated neurite outgrowth induced by anti-PirB ( $\mathrm{P}<0.05 ;$ Fig. $4 \mathrm{~A}$ and $\mathrm{B})$. In addition, western blot analysis demonstrated that the protein expression of several axon outgrowth-associated genes also decreased in the presence of either LY294002 or rapamycin (Fig. 4C). These data strongly confirm that PirB exerts inhibitory effects on axonal growth through the PI3K/Akt/mTOR pathway.

\section{Discussion}

Accumulating evidence suggests that the lack of regeneration capacity in the adult mammalian CNS is due to the presence of certain myelin-derived inhibitors, including the repulsive trio (Nogo-66, MAG and OMgp) $(4,5)$. Inhibitory signaling is transduced via their receptors, $\mathrm{NgR}$ and PirB. The trimeric receptor complexes stimulate the RhoA-Rho-associated protein kinase (ROCK) pathway when myelin-associated inhibitors bind with Nogo-A. It is reported that PirB may have a role in inhibiting axonal regeneration in specific PirB-expressing neurons in the CNS (15). However, the molecular mechanism underlying PirB mediated inhibition of axonal outgrowth remains unclear. In the present study, 


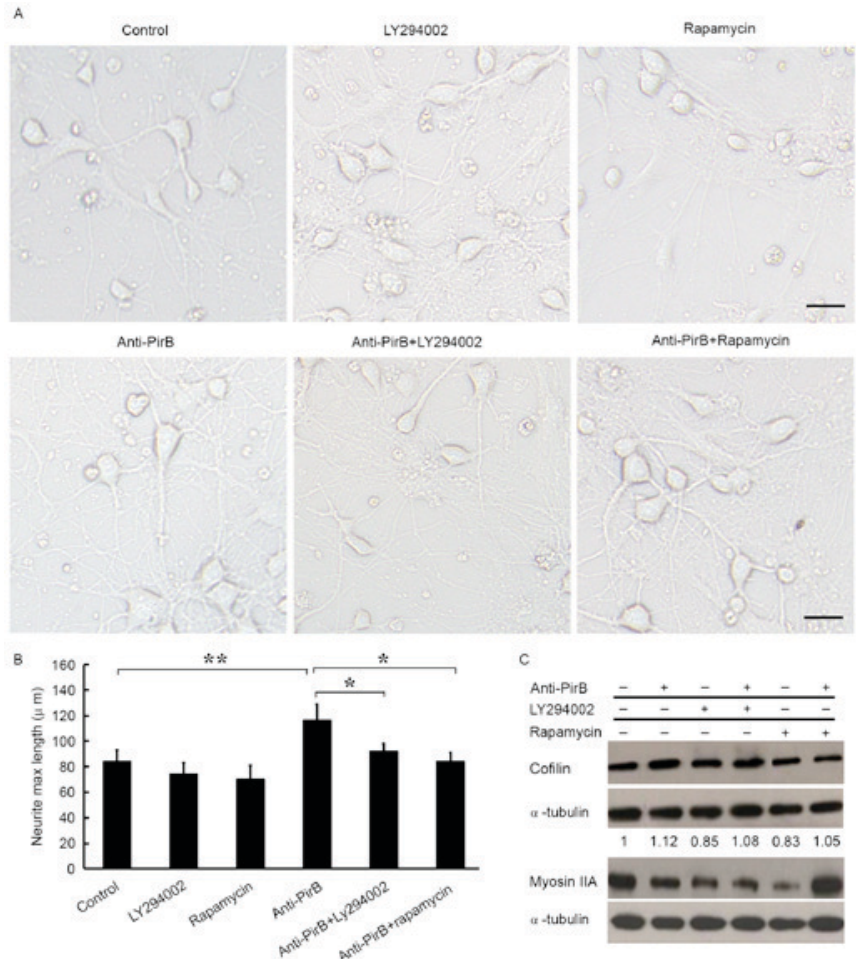

Figure 4. Blockade of the PI3K/Akt/mTOR pathway reduced Anti-PirB-induced stimulatory effects on axonal outgrowth in cultured neurons with Nogo-66. (A) Effect of a PI3K inhibitor (LY294002; $50 \mu \mathrm{mol} / \mathrm{l}$ ) or rapamycin (an mTOR inhibitor; $100 \mathrm{nmol} / \mathrm{l}$ ) on axonal outgrowth. (B) Effects of Anti-PirB together with either LY294002 or rapamycin on neurite length. (C) Effects of Anti-PirB together with either LY294002 or rapamycin on the axonal outgrowth associated-protein expression. ${ }^{*} \mathrm{P}<0.05$, ${ }^{* *} \mathrm{P}<0.01$. Scale bar, $20 \mathrm{~mm}$. PirB, leukocyte immunoglobulin like receptor B1; PI3K, phosphoinositide 3-kinase; Akt, Akt serine/threonine kinase; mTOR, mechanistic target of rapamycin kinase.

PirB expression was induced by Nogo-66 in cortical neurons isolated from newborn mice. Subsequently, PirB antibodies were used to block the effect of PirB to determine how axonal regeneration was affected. The results of the current study indicate that the PirB-mediated inhibitory effects are mediated via $\mathrm{PI} 3 \mathrm{~K} / \mathrm{Akt} / \mathrm{mTOR}$ signaling pathway.

The inhibitory effects of PirB on axonal outgrowth have been demonstrated. Attenuation of PirB activity through antibody antagonism or genetic approaches can partially reduce the inhibition of neurite outgrowth in vitro and in vivo (9). PirB suppresses axonal outgrowth via SH3 domain containing ring finger 1 (POSH)/ROCK or and POSH/c-Jun N-terminal kinases pathway (16). Furthermore, the substrates of tropomyosin receptor kinase $\mathrm{B}(\operatorname{TrkB})$ include tyrosine-kinase and mitogen-activated protein kinase signaling complexes, such as PI3K and Akt, ultimately leading to inhibition axonal regeneration (9). PirB suppresses axon regeneration through inhibition of Trk activity (17). The Trk receptor stimulates PI3K heterodimers, which leads to the activation of pyruvate dehydrogenase kinase 1 and Akt (18), subsequently activating mTOR pathway. However, the effect of PirB on PI3K/Akt/mTOR on axonal outgrowth remains poorly understood. The present study aimed to examine the effects of PirB on axonal outgrowth in the presence $\mathrm{NgR}$ inhibition and explored the mechanism of PirB-inhibited axonal outgrowth via PI3K/Akt/mTOR pathway.
The results of the current study showed that increased $\mathrm{PI} 3 \mathrm{~K} / \mathrm{Akt} / \mathrm{mTOR}$ activity in the cortical neurons of newborn mice induced by Nogo-66. This finding suggests that $\mathrm{PI} 3 \mathrm{~K} / \mathrm{Akt} / \mathrm{mTOR}$ is regulated by $\mathrm{PirB}$ and that $\mathrm{PI} 3 \mathrm{~K} / \mathrm{Akt} / \mathrm{mTOR}$ may be the signaling pathway that transduces inhibitory signaling from PirB to the intracellular part of neurons. PirB inhibited Trk activity in process of suppressing axon regeneration (17). The inhibitory influence of exogenous PirB on axonal outgrowth of cortical neurons induced by Nogo-66 was investigated in the present study. Compared with the control groups, anti-PirB treatment promoted axonal outgrowth. Furthermore, myosin IIA and cofilin protein expression was increased in following anti-PirB treatment in cortical neurons from newborn mice induced by Nogo-66. Taken together, results from our studies support the hypothesis that PirB has an important role in inhibiting axonal outgrowth through PI3K/Akt/mTOR.

PI3K signaling cascades are important regulators invovled in a host of cellular activities such as apoptosis, proliferation, differentiation and axonal outgrowth (19). In mammals, PI3K/Akt/mTOR signaling pathway has a crucial role in regulating axonal outgrowth (11). This study revealed that PirB exerted the inhibitory effects through the PI3K/Akt/mTOR signaling pathways. Nogo-66 treatment induced activation of the PI3K/Akt/mTOR signaling in cortical neurons of newborn mice in the presence of $\mathrm{NgR}$ blockade, as determined by quantitying the expression of PI3K, Akt1, Akt2 and mTOR PI3K/Akt/mTOR pathway. Phosphorylated Akt induced by PI3K can increase accumulation and activation of the mTOR-raptor kinase complex, subsequently mediating phosphorylation of S6 kinase to enhance protein synthesis (20). Furthermore, the pharmacological inhibitors of PI3K and mTOR (LY294002 and rapamycin) were used to confirm the involvement of PI3K/Akt/mTOR signaling pathway in PirB-mediated inhibition of axonal outgrowth in cortical neurons of newborn mice. The results indicated that activation of the PI3K/Akt/mTOR pathway significantly reversed the inhibitory effects of PirB on axonal outgrowth, since the blocked factors by PirB were attenuated in the presence of either of two pharmacological inhibitors, as determined by neurite outgrowth assay and measuring the mRNA levels and protein associated with axonal outgrowth and regulation of the PI3K/Akt/mTOR pathway. These findings confirm that the PI3K/Akt/mTOR signaling pathway is involved in PirB-mediated inhibition of axonal outgrowth.

In conclusion, the findings of the current study suggest that PirB exerts its inhibitory effects on axonal outgrowth through PI3K/Akt/mTOR signaling pathway. These findings will provide a novel insight into understanding the role of PirB in axonal outgrowth and the molecular mechanisms of PirB signaling, thus, potentially contributing to the development of novel therapeutic strategies to encourage axonal regeneration following CNS injury.

\section{Acknowledgements}

The present study was granted from the Youth Project of Shanghai municipal Commission of Health and Family Planning (grant no. 20144Y0229). 


\section{References}

1. Pernet V and Schwab ME: Lost in the jungle: New hurdles for optic nerve axon regeneration. Trends Neurosci 37: 381-387, 2014.

2. Shum JW, Liu K and So KF: The progress in optic nerve regeneration, where are we? Neural Regen Res 11: 32-36, 2016.

3. Li S, Yang C, Zhang L, Gao X, Wang X, Liu W, Wang Y, Jiang S, Wong $\mathrm{YH}$, Zhang $\mathrm{Y}$ and Liu K: Promoting axon regeneration in the adult CNS by modulation of the melanopsin/GPCR signaling. Proc Natl Acad Sci USA 113: 1937-1942, 2016.

4. Rao SN and Pearse DD: Regulating axonal responses to injury: The intersection between signaling pathways involved in axon myelination and the inhibition of axon regeneration. Front Mol Neurosci 9: 33, 2016.

5. Wang KC, Koprivica V, Kim JA, Sivasankaran R, Guo Y, Neve RL and He Z: Oligodendrocyte-myelin glycoprotein is a Nogo receptor ligand that inhibits neurite outgrowth. Nature 417: 941-944, 2002.

6. Domeniconi M, Cao Z, Spencer T, Sivasankaran R, Wang K, Nikulina E, Kimura N, Cai H, Deng K, Gao Y, et al: Myelin-associated glycoprotein interacts with the Nogo66 receptor to inhibit neurite outgrowth. Neuron 35: 283-290, 2002.

7. Fournier AE, GrandPre T and Strittmatter SM: Identification of a receptor mediating Nogo-66 inhibition of axonal regeneration. Nature 409: 341-346, 2001.

8. Atwal JK, Pinkston-Gosse J, Syken J, Stawicki S, Wu Y, Shatz C and Tessier-Lavigne M: PirB is a functional receptor for myelin inhibitors of axonal regeneration. Science 322: 967-970, 2008.

9. Gou Z, Mi Y, Jiang F, Deng B, Yang J and Gou X: PirB is a novel potential therapeutic target for enhancing axonal regeneration and synaptic plasticity following CNS injury in mammals. J Drug Target 22: 365-371, 2014.
10. Liu J, Wang Y and Fu W: Axon regeneration impediment: The role of paired immunoglobulin-like receptor B. Neural Regen Res 10: 1338-1342, 2015.

11. Wang $\mathrm{H}$, Xiong $\mathrm{Y}$ and $\mathrm{Mu} \mathrm{D}$ : PirB restricts neuronal regeneration in developing rat brain following hypoxia-ischemia. Mol Med Rep 6: 339-344, 2012.

12. Abe N, Borson SH, Gambello MJ, Wang F and Cavalli V: Mammalian target of rapamycin (mTOR) activation increases axonal growth capacity of injured peripheral nerves. J Biol Chem 285: 28034-28043, 2010.

13. Deng Q, Cai W, Li S, Zhang Y and Su B: Small Nogo-66-binding peptide promotes neurite outgrowth through RhoA inhibition after spinal cord injury. Brain Res Bull 99: 140-144, 2013.

14. Livak KJ and Schmittgen TD: Analysis of relative gene expression data using real-time quantitative PCR and the 2(-Delta Delta C(T)) method. Methods 25: 402-408, 2001.

15. Nakamura Y, Fujita Y, Ueno M, Takai T and Yamashita T: Paired immunoglobulin-like receptor B knockout does not enhance axonal regeneration or locomotor recovery after spinal cord injury. J Biol Chem 286: 1876-1883, 2011.

16. Rusanescu G, Yang W, Bai A, Neel BG and Feig LA: Tyrosine phosphatase SHP-2 is a mediator of activity-dependent neuronal excitotoxicity. EMBO J 24: 305-314, 2005.

17. Dickson HM, Zurawski J, Zhang H, Turner DL and Vojtek AB: POSH is an intracellular signal transducer for the axon outgrowth inhibitor Nogo66. J Neurosci 30: 13319-13325, 2010.

18. Fujita Y, Endo S, Takai T and Yamashita T: Myelin suppresses axon regeneration by PIR-B/SHP-mediated inhibition of Trk activity. EMBO J 30: 1389-1401, 2011.

19. Segal RA: Selectivity in neurotrophin signaling: Theme and variations. Annu Rev Neurosci 26: 299-330, 2003.

20. Engelman JA, Luo J and Cantley LC: The evolution of phosphatidylinositol 3-kinases as regulators of growth and metabolism. Nat Rev Genet 7: 606-619, 2006. 